\title{
Linear and Circular Dichroism in Graphene-Based Reflectors for Polarization Control
}

\author{
M. Amin $\odot,{ }^{1,}{ }^{*}$ O. Siddiqui, ${ }^{1}$ and M. Farhat ${ }^{2}$ \\ ${ }^{1}$ College of Engineering, Taibah University, Madinah 41411, Saudi Arabia \\ ${ }^{2}$ Division of Computer, Electrical, and Mathematical Science and Engineering, King Abdullah University of \\ Science and Technology, Thuwal 23955-6900, Saudi Arabia
}

(Received 18 October 2019; revised manuscript received 10 January 2020; accepted 31 January 2020; published 19 February 2020)

\begin{abstract}
We present an ultrathin graphene metascreen that possesses dispersive optical activity in the early terahertz spectrum. The metascreen design consists of periodically etched L-shaped voids on a graphene substrate backed by a conductive plane. The specific unit-cell design is based on chirality and leads to highly asymmetric radiations from the plasmon-polariton surface currents, leading to linear and circular dichroism. Hence the incident linearly or circularly polarized electric fields are effectively absorbed by the metasurface in different proportions. Consequently, the metasurface assumes half- and quarter-waveplate behaviors in different parts of the reflected optical spectrum. In particular, we show via full-wave simulations that the dichroic metascreen supports perfect linear-to-circular polarization conversion (circular dichroism) in two adjacent terahertz frequency bands. In two other terahertz bands, it rotates the incoming linearly polarized wave vector by $90^{\circ}$ (linear dichroism). Moreover, since graphene has a variable refractive-index dependence on its chemical potential, the dispersion characteristics can be shifted to neighboring frequencies within the early terahertz spectrum. We further demonstrate an angularly stable response for incident angles varying between $0^{\circ}$ and $45^{\circ}$. The tunable linear and circular dichroism characteristics are well suited for applications in sensing, imaging, and spectroscopy at terahertz frequencies.
\end{abstract}

DOI: 10.1103/PhysRevApplied.13.024046

\section{INTRODUCTION}

The transverse electromagnetic nature allows light waves to be characterized in terms of the orientation of the associated electric field vectors, commonly known as the polarization. The polarization state of a light wave can take different forms such as linear, circular, or elliptical depending on the progression of the electric field vector in the time domain [1,2]. From a practical viewpoint, information can be concealed in the polarization states without varying the intensity of the associated light wave; or, by decrypting the polarization state of the outgoing wave, the material properties can be estimated. We can identify several applications in daily life where the polarization state of light has been exploited, such as optical displays, spectroscopy, and optical antennas [1-3]. Many traditional polarization control systems employ either birefringent crystals or Faraday rotators to change the state of the electric field orientation [4-6]. However, both of these optical systems have large device footprints, making their use difficult for realistic applications. Additionally, Faraday rotation consumes a large

\footnotetext{
*masharif@taibahu.edu.sa
}

amount of power, which is undesirable in modern optical devices. Dichroism is one of those natural mechanisms of polarization control that involves absorption of specific linear polarizations with light transmission [1,7]. An example is the dichroic herapathite crystal, which was used in the earlier Polaroid sheets [1]. Some crystalline structures having circular dichroism rotate the electric field polarization of the incident light, a property known as optical activity. While linear dichroism is based on the absorption of linear components, circular dichroism (CD) introduces an absorption differential between the left- and right-handed circular polarizations. Hence circular or elliptical polarized light emerges from circularly dichroic materials. Circular and linear dichroism are natural ways to control polarized light but since they are based on weak wave interactions, the resulting structures have high-profile geometries that are hard to incorporate with modern integrated circuits [4]. Therefore, there is a vital requirement for an opticalpolarization-control method to be passive (unlike Faraday rotation) and low profile, so that device integration at subwavelength scale can be achieved. Taking these practical bounds into account, electromagnetic metasurfaces have been suggested as efficient polarization-control alternatives [8-13]. Metasurfaces have also been designed at 
microwave, terahertz $(\mathrm{THz})$, and optical frequencies for various other applications ranging from perfect absorbers [14-16] to sensors [17,18], thermal emitters [19], and imaging devices $[20,21]$. The subwavelength size of the unit cell in the case of metasurfaces is essential for neardiffraction-limit imaging and wide-angle beam-steering applications [22,23]. These thin-film metasurfaces are designed using metallic radiating elements to manipulate electromagnetic waves [9-13,24-26]. The planar twodimensional (2D) metasurface counterparts of the traditional polarizers are relatively easier to design, fabricate, and characterize. These passive structures are inspired by optically active materials and crystals, which exhibit optical rotation due to the inherent chirality in their constitutive structure, which renders refractive surfaces represented by the following dielectric tensor [3]:

$$
\overline{\bar{\varepsilon}}=\left[\begin{array}{ccc}
\varepsilon_{1} & i a & 0 \\
-i a & \varepsilon_{1} & 0 \\
0 & 0 & \varepsilon_{3}
\end{array}\right] .
$$

Hence a wave propagating in the $z$ direction would then have its $x$ - and $y$-field components oscillate with a $\pi / 2$ phase difference, rendering a circularly polarized wave. The analogous behavior in the chiral metasurface is obtained by designing unit cells that are mirror asymmetric or geometrically chiral.

The motivation of this paper follows from Fig. 1, which shows a comparison between the two principles of optical rotation, i.e., the birefringent rotary system and its chiral metasurface analog in transmission mode. Since the optical axis is tilted by $45^{\circ}$ with respect to the incoming linear polarization, the birefringent crystal splits the input polarization into ordinary and extraordinary waves, which travel at different velocities and hence emerge with a $\pi / 2$ phase difference, leading to circular polarization. On the other hand, the circular polarization for a chiral metasurface is obtained because of two underlying mechanisms. Due to the highly asymmetric unit-cell structure, high-intensity retarded currents result on the two axes of the metallic inclusion at the plasma resonance. The radiation from these currents adds constructively, with various phase differences in the far field region to create a certain polarization state. Hence linearly polarized incident waves are converted to cross- or circularly polarized scattered waves, depending on the dispersion relation [27]. Since the optical activity is derived from resonant unit cells, the latest challenge is to extend the chiralmetasurface designs that operate over broadband spectra into the microwave and optical regimes [28,29]. Additionally, a dramatic improvement would be dynamic control of the operating frequencies, which has been done previously with tunable electromagnetic materials [30,31].

Among the reconfigurable artificial materials, graphenebased structures have recently gained popularity [32-34].
Graphene is a natural contender for tunable metasurfaces, primarily due to the fact that it is possible to vary its electrical properties directly by controlling the electric potential [35]. The other metamaterial structures, on the other hand, rely on external components such as inductors, capacitors, and diodes to achieve reconfigurability. Since graphene was first isolated in 2004 [36], significant studies have been carried out that highlight its interesting physical parameters and technical and scientific applications in various areas, e.g., mechanics, chemistry, and biology, as well as in photonics and electronics [37]. Graphene has a truly 2D structure formed by carbon atoms, resulting in an ultrathin profile known as a monolayer. Additionally, the graphene material has stable thermal properties, ultrahigh carrier mobility, and an electrical conductivity that is dependent on the density of the carrier, with a plasma frequency in the terahertz and infrared (IR) regimes [38]. Graphene can also replace noble metals such as gold and silver, which suffer from high conductive losses in the optical regime.

Exploiting these unique properties, we present in this paper a chiral graphene metasurface designed by exploiting two fundamental mechanisms discussed above in reflection mode. The geometrical chirality that renders a dichroic structure (as in CD crystals) is obtained by etching mirror-asymmetric unit cells on the graphene monolayer, such that the structure supports the surface-plasmonpolariton (SPP) pair when interfaced with a dielectric material. SPPs are strong resonances, the operating frequency of which is a function of the unit-cell dimensions, the permittivity of the dielectric, and graphene's Fermi level $[39,40]$. Compared to their metallic counterparts, graphene SPPs are better confined (small footprint), propagate for longer, and are frequency reconfigurable by chemical doping in the terahertz range [35]. Recently, graphene-based tunable cross-polarizers have been proposed to transform incident polarization to cross-polarized fields $[41,42]$. The unit cells of these structures have been formed by isolated graphene patches, which have had to be separately biased. Our unit cell is fundamentally different, as it is a complementary L-shaped structure obtained by creating voids on continuous graphene layers. This simple (yet powerful) fabrication technique renders a structure that is continuously conducting, so that the graphene cells can be biased with a greatly simplified dc network consisting of a single gate-voltage source. This dynamic tunability leads to a full range of polarization-state conversion (circular to linear) that has not been reported in previous works $[41,42]$. We show via full-wave simulations that the designed chiral metasurface reflector is optically active in the early terahertz frequency range, with a variety of polarization-conversion possibilities. In particular, we demonstrate the half- and quarter-wave-plate behaviors in a tunable broadband terahertz spectrum, where the polarization-conversion ratio (PCR) remains above the 
(a)

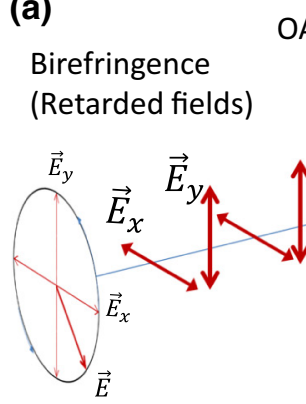

(b)

Chiral metasurface

(Radiation from asymmetric currents distributions)

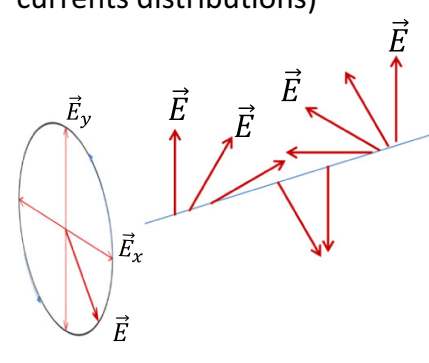

Polarization conversion (quarter wave plate)

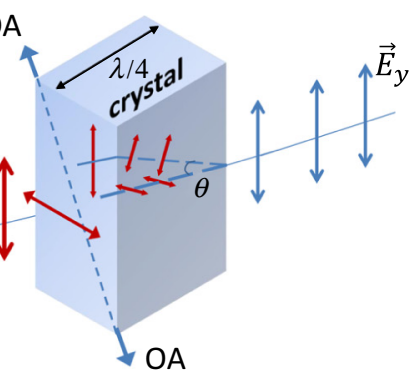

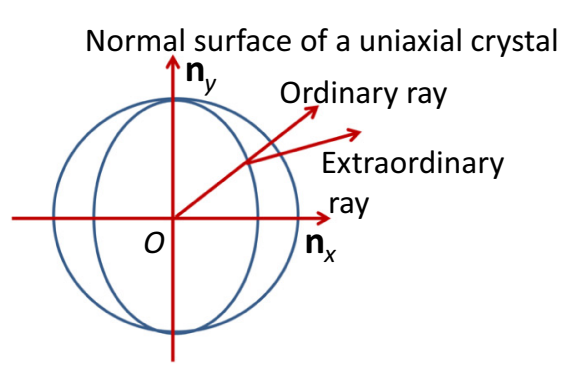

Polarization control using birefringence

bin (c)

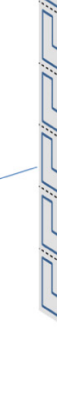

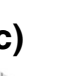
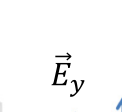

Polarization control exploiting chiral metasurfaces

FIG. 1. Two mechanisms of optical polarization control are depicted. (a) Linear-tocircular polarization conversion using the birefringence of a uniaxial crystal, the refractive-index surface of which is depicted on the right. The optical axis (OA) is tilted by $45^{\circ}$ with respect to the incoming polarization. The extraordinary ray is slower than the ordinary ray so that the $x$ and $y$ electric fields have a phase difference of $90^{\circ}$, leading to circular polarization. (b) The circularly polarized fields on the other side of the metasurface are formed by the superposition of radiation from retarded asymmetric currents owing to the chirality of the constituent unit cells.
$90 \%$ threshold. The electro-optic tunability allows realtime control of this unique optical-activity behavior by applying a gate voltage to the graphene layer.

\section{DESIGN OF THE DICHROIC GRAPHENE METASCREEN}

Classical plasmonics has been facing many problems and limitations in recent years due to interband transitions at IR and optical frequencies that increase the losses and also due to the fixed value of the plasma frequency, which lacks reconfigurability [43]. Some attempts have been put forward to make use of semiconductors, such as transparent conductive oxides (TCOs) or metal nitrides, to design tunable optical devices in the near-IR and IR ranges [44]. However, these semiconductors are difficult to manufacture and exhibit considerable losses in the mid-IR and terahertz ranges. On the other hand, graphene materials have been shown to solve many of these issues, due to their low losses and their tunable optical response [45]. In recent years, a plethora of terahertz and IR graphene components have been devised, including waveguides and plasmonic interconnects [46], sensors [47], modulators [31], perfect absorbers [48-50], couplers [51], and photomixing frequency synthesizers [52]. The achievement of these capabilities at the nanoscale, with the potential for frequency reconfiguration, could pave the way for sensing and communication networks with carrier frequencies up to the terahertz frequencies and well into the IR spectrum. The proposed graphene-based tunable chiral metasurfaces have the potential to enhance the capabilities of existing optical sensing and spectroscopic techniques. For example, the polarization states (determined for the reflected fields) intrinsically take into account the wave propagation inside the sample for the orthogonal electric field components. Hence, by exploiting the chirality, graphene-based terahertz sensors [53-55] may be further improved in order to detect the dielectric properties of anisotropic materials. Similarly, the surfaceplasmon modes in graphene-based terahertz spectroscopy are found to be well suited for detection of the vibrational modes of molecules [56]. Adsorption in graphene nanoribbons offers measurable dips in extinction spectrum due to gas concentration changes, which may be employed in gas-molecule sensing [57]. Considering the highly dispersive and frequency-selective chiral modes observed in the proposed metasurface, polarization-state detection can also provide a sensitive alternative to the existing surfaceplasmon method. Furthermore, the proposed structures are spectrally tunable, leading to a broadband chiral response, which is a desirable property in spectroscopic applications.

In what follows, we present the design of a circularly chiral reflecting metasurface that is based on exciting the SPP resonances on the graphene-dielectric interface. To achieve this in practice, the material is etched out from the graphene monolayer to form a periodic arrangement of $L$-shaped voids on its surface, as shown in Fig. 2. The advantage of creating voids instead of depositing is to 


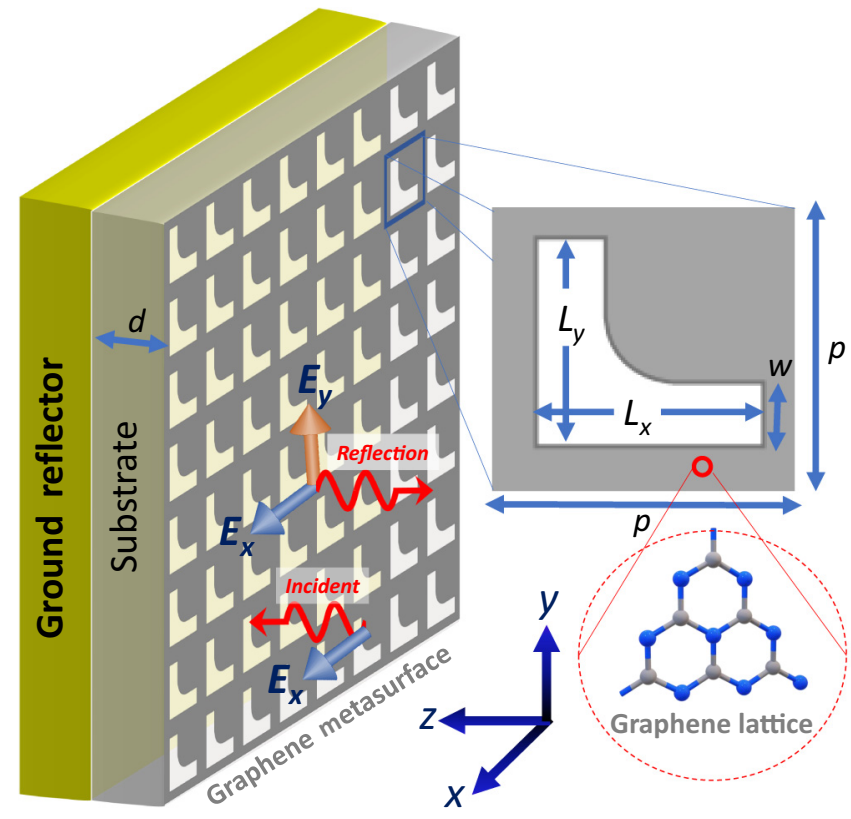

FIG. 2. A schematic illustration of a graphene metascreen consisting of an atomic layer of graphene engraved with a periodic array of L-shaped etched voids. The inset shows a schematic representation of the unit cell and the atomic scale graphene lattice.

obtain a continuous graphene surface, so that the biasing can be done by a single gate-voltage source. According to Babinet's principle, such a geometry would just interchange the role of the electric and magnetic fields, without affecting the chiral behavior [58]. The graphene surface resides on a thin substrate of thickness $d=15 \mu \mathrm{m}$ and is backed up by a metallic ground plane (a perfect electric conductor) that serves as a fully reflecting surface.

This specific design serves two purposes. First, the interface between the graphene and the dielectric supports the formation of SPPs on the graphene monolayer, which propagate as surface waves in the $x-y$ plane [43]. Second, the unique diagonal symmetry of the unit cell allows the generation of helicoidal fields in the L-shaped void, leading to the optical activity. The arms of the unit-cell voids ( $L_{x}$ and $L_{y}$ as outlined in Fig. 2) have dimensions of 7.5 $\mu \mathrm{m}$ and the width of the arm (w) measures $1 \mu \mathrm{m}$ such that the period of the structure is $p=10 \mu \mathrm{m}$. Therefore, the unit-cell size is ultracompact, measuring around $\lambda / 15$ at $2 \mathrm{THz}$, and is quite suitable for the on-chip integration in modern optical systems.

Note in Fig. 2 the curved part at the intersection of the two arms, which is designed to reduce spurious radiations due to sharp corners. A unique feature of the dichroic metasurface is its polarization-independent reflection response, which is due to the diagonally symmetric shape of the unit cell. This can be noted well in Fig. 2 for a normally incident plane wave, where the two incident polarizations are treated equally by the unit cells. A relative permittivity of $r=4$ is used for the dielectric substrate. The frequencydependent complex permittivity $\left(\epsilon_{g}\right)$ of graphene is determined from the surface conductivity $\left(\sigma_{g}\right)$ :

$$
\varepsilon_{g}(\omega)=1+\frac{j \sigma_{g}(\omega)}{\varepsilon_{0} \omega \Delta}
$$

Here, $\epsilon_{0}$ is the free-space permittivity, $\omega$ is the angular frequency, and $\Delta=1 \mathrm{~nm}$ is the thickness of the graphene layer.

At terahertz frequencies, the surface conductivity of graphene $\left(\sigma_{g}\right)$ is typically calculated from Kubo's formula. Here, the conductivity is modeled by electron-hole pair excitations due to contributions from inter- and intraband transitions within the graphene layer. At low frequencies, interband transitions are negligible and the conductivity is mainly due to intraband transitions, as follows:

$\sigma_{g}(\omega)=\frac{i e_{0}^{2} k_{B} T}{\pi \hbar(\hbar \omega+i \Gamma)}\left\{\frac{\mu_{c}}{k_{B} T}+2 \log \left[\exp \left(\frac{-\mu_{c}}{k_{B} T}\right)+1\right]\right\}$.

Here, $k_{B}$ is the Boltzmann constant, $T$ is the temperature on the kelvin scale, $e_{0}$ is the electron charge, $\hbar$ is the reduced Planck's constant, $\Gamma$ is the damping coefficient, and $\mu_{c}$ is the chemical potential of the graphene layer. The chemical potential can be varied by chemical doping or by applying a gate-voltage bias to the graphene sheet. The damping coefficient $\Gamma$ can be calculated as follows:

$$
\Gamma=-\left(e_{0} \hbar v_{f}^{2}\right) /\left(\mu \mu_{c}\right)
$$

where the constants $v_{f}=10^{6} \mathrm{~m} / \mathrm{s}$ and $\mu=100,000 \mathrm{~cm}^{2} /$ $\mathrm{Vs}$ are the Fermi velocity and the electron mobility, respectively.

\section{JONES-MATRIX ANALYSIS}

In this section, we demonstrate the polarization changes in the reflection spectrum of the graphene metascreen when a linearly polarized electric fields is incident on it. The two types of dichroism, i.e., the linear to linear conversion and the linear-to-elliptical conversion, which can be governed by the chemical potential of the graphene layer, are described in Fig. 3. Before presenting the simulation results, it is instructive to describe the relationship between the incident fields and the reflected fields in terms of the Jones matrices. Figure 3 explains the two possible conversion mechanisms for an $x$-directed incident field. For the generalized case, $E_{x i}$ and $E_{y i}$ can be assumed as the incident electric field components along the $x$ and $y$ axes, respectively, with $E_{x r}$ and $E_{y r}$ as the reflected electric field components. The Jones matrix of the reflected parameters 

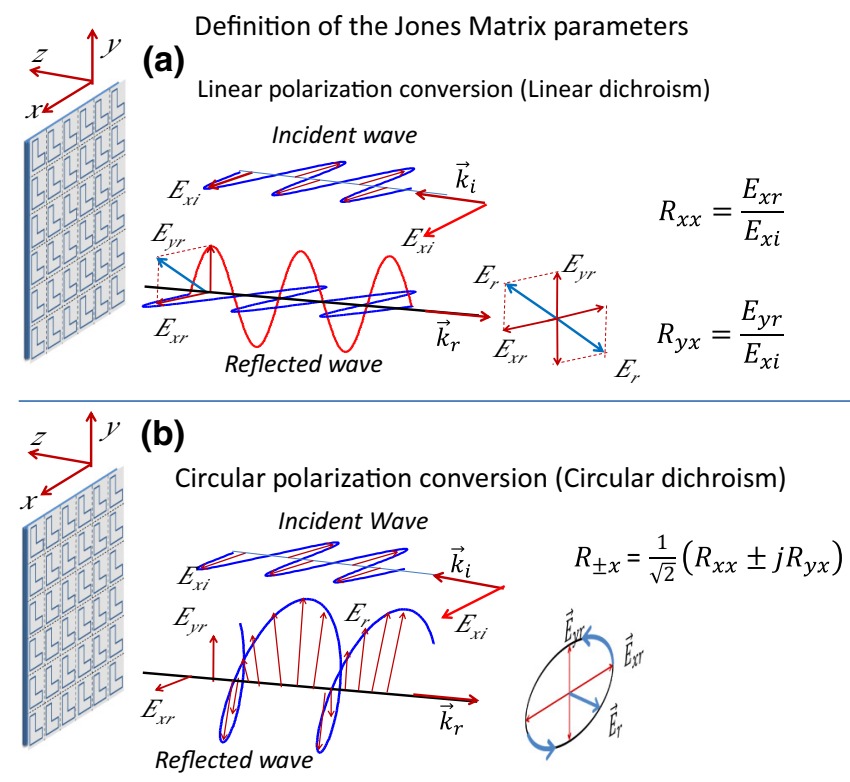

FIG. 3. A demonstration of the Jones-matrix parameters for the two reflection geometries. (a) The definition of the linearpolarization reflection coefficients for an $x$-polarized incident wave. (b) The Jones-matrix definition in circular bases. The reflected wave can be considered as a combination of two circularly polarized waves rotating in opposite directions.

can then be described by the following relationship:

$$
\left(\begin{array}{c}
E_{x r} \\
E_{y r}
\end{array}\right)=\left(\begin{array}{ll}
R_{x x} & R_{x y} \\
R_{y x} & R_{y y}
\end{array}\right)\left(\begin{array}{c}
E_{x i} \\
E_{y i}
\end{array}\right)
$$

where $R_{x x}$ and $R_{y x}$ are the co- and cross-polarized reflection coefficients for the $x$-polarized incident fields, while $R_{x y}$ and $R_{y y}$ are the corresponding reflection parameters for the $y$-polarized incident fields. The reflection coefficients in circular bases can be written in the form of the linear coefficients in the following manner:

$$
R_{ \pm x}=\frac{1}{\sqrt{2}}\left(R_{x x} \pm j R_{y x}\right), \quad R_{ \pm y}=\frac{1}{\sqrt{2}}\left(R_{x y} \pm j R_{y y}\right)
$$

In the Jones calculus, the conversion of linearly polarized incident waves into elliptically polarized reflected waves can then be represented by the following equation:

$$
\left(\begin{array}{c}
E_{+r} \\
E_{-r}
\end{array}\right)=\left(\begin{array}{ll}
R_{+x} & R_{+y} \\
R_{-x} & R_{-y}
\end{array}\right)\left(\begin{array}{c}
E_{x i} \\
E_{y i}
\end{array}\right)
$$

Here, the subscript $+x$ and $-x$ signs represent the rightand left-handed components of the reflected waves, respectively. Referring to Eqs. (6) and (7) and considering Fig. 3 for $x$-polarized incidence, it can be readily deduced that a pure circularly polarized reflected wave is possible only if the $x$ and $y$ components have equal magnitudes, $\left(\left|E_{x r}\right|=\left|E_{y r}\right|\right)$, with a phase difference of $\pm 90^{\circ}$.
Next, we characterize our graphene metascreen by determining the elements of the linear Jones matrix of Eq. (5) with variation of the chemical potential of graphene. The full-wave simulations are performed using the finiteelement-method- (FEM) based electromagnetic software COMSOL. Figure 2 shows the simulation domain, in which the unit cell is terminated by the periodic boundary conditions along the $x$ and $y$ directions. The negative $z$ direction is terminated by a perfect matched layer (PML) to prevent spurious reflections originating from boundaries. As shown in Fig. 3, the metascreen is illuminated by an $x$-polarized electromagnetic wave to simplify the demonstration of the reflected wave's polarization states. Hence, with the electric field oriented along one of the transverse planes, we can fully characterize the metascreen by determining two of the four Jones reflection parameters (i.e., $R_{x x}$ and $R_{y x}$. The conductivity of the graphene is varied by changing the chemical potential between 300 and 800 meV according to Eq. (3). A relative permittivity $\left(\epsilon_{r}\right)$ of 4 and a height $(d)$ of $15 \mu \mathrm{m}$ are used for the dielectric substrate. The resulting reflection coefficients are displayed as surface-intensity plots in Figs. 4(a) and 4(b) over a wide range of terahertz spectrum. With the increase in the chemical potential, a blue shift in the metalayer's resonance is noted in the surface plot, which is consistent with the direct relation between graphene's Fermi level and the plasma resonances. Note the high optical activity in the region between the half-power points, indicated on the reflectance-surface plots (Fig. 4) at the $600-\mathrm{meV}$ potential threshold. In this region, considerable reflected power is also carried by the $y$-polarized wave. In particular, consider the two 3-dB points on which the $x$ - and $y$-directed fields contribute equally to the reflected power, which is one of the conditions leading to circularly polarized waves.

For a deeper look at the polarization-conversion phenomenon, the magnitude and phase of the reflection coefficients $R_{x x}$ and $R_{y x}$ are extracted for a chemical potential of $600 \mathrm{meV}$ and are depicted in Figs. 4(c) and 4(d). A broadband region of high cross-polarization conversion is observed between 1.94 and $2.68 \mathrm{THz}$. Since the incident fields are purely $x$-polarized, the $100 \% y$-polarized reflectance at some frequencies satisfies the half-waveplate condition. Similarly, at the two 3-dB points, which are identified by the crossing of the two orthogonal components of the electric field (1.85 and $2.8 \mathrm{THz})$, the phase difference is approximately $90^{\circ}$, which can lead to circularly polarized waves (see the next section). Finally, the near-field distribution in the L-shaped unit cells given in Fig. 4(d) may shed light onto the mechanism of resonance formation. At $2.04 \mathrm{THz}$, the predominantly $y$-oriented electric field vectors in the lower slot show high crosspolarization conversion. On the other hand, at $2.81 \mathrm{THz}$, intense electric field vectors are present in both orthogonal directions in the upper and lower slots, leading to the 3-dB condition. 


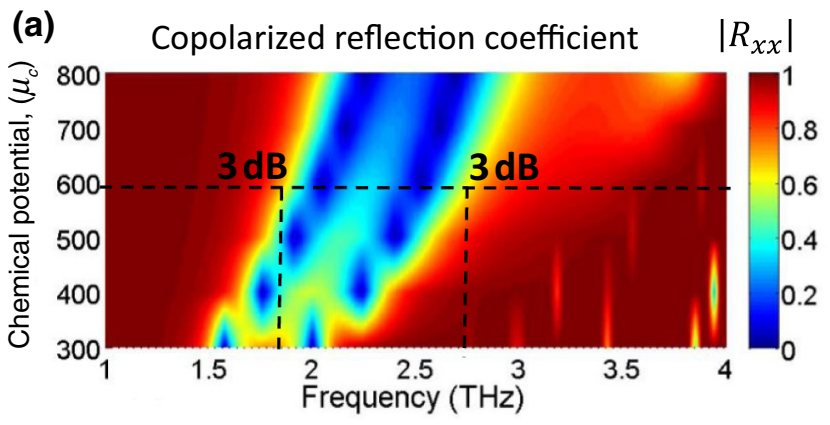

(c) $\quad\left|R_{x x}\right|$ and $\left|R_{y x}\right|$ for $\mu_{c}=600 \mathrm{meV}$

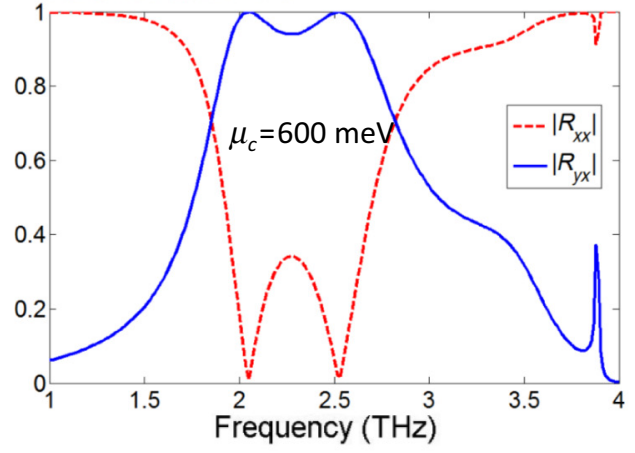

(d) Reflection phases for $\mu_{c}=600 \mathrm{meV}$

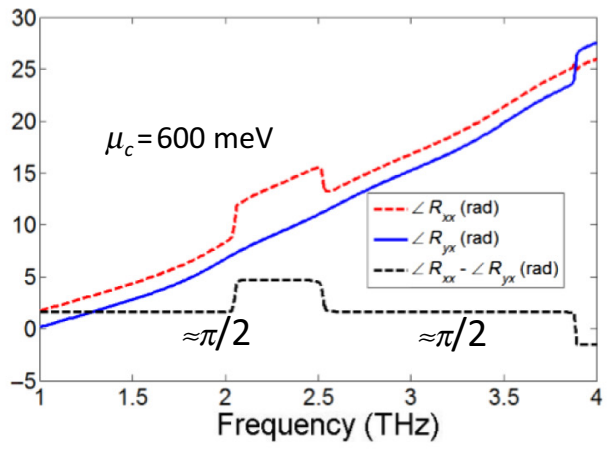

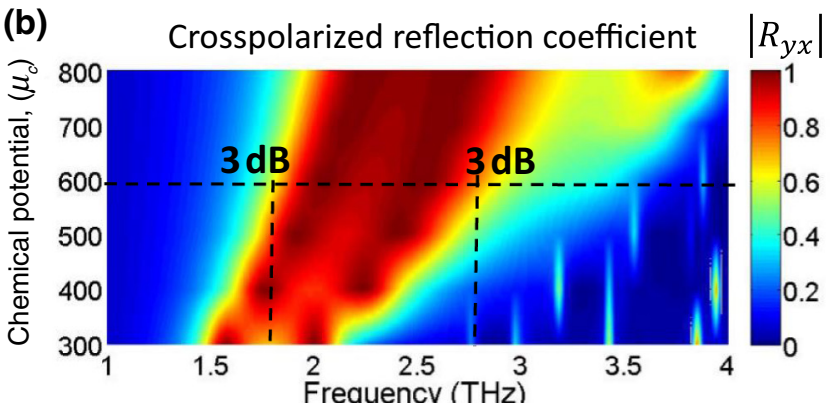

(e) Unit cell Electric fields

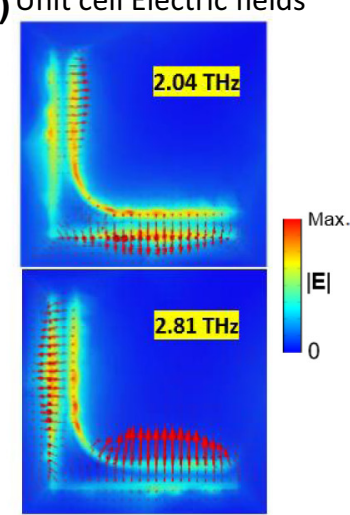

FIG. 4. (a) The amplitude of the copolarized reflection coefficients $\left|R_{x x}\right|$ when the chemical potential $\left(\mu_{c}\right)$ is varied between 300 and $800 \mathrm{meV}$. (b) The amplitude of the cross-polarized $\left|R_{y x}\right|$ due to a similar variation in the chemical potential. (c) The spectral response of the magnitudes of the reflection coefficients extracted from (a) and (b) for a chemical potential of $600 \mathrm{meV}$. (d) The phases of the two reflection coefficients and their difference $\angle R_{x x}-\angle R_{y x}$, also obtained from (a) and (b). (e) The electric field distribution observed in the near field of the graphene metascreen at two frequencies, 2.04 and $2.81 \mathrm{THz}$, where the polarization states are linear and circular, respectively. The arrows indicate direction and the color plot represents the intensity of the near electric field.

\section{QUARTER-AND HALF-WAVE-PLATE BEHAVIORS IN THE GRAPHENE METASCREEN}

Two extreme outcomes of dichroism (as highlighted earlier) are the half- and quarter-wave-plate behaviors of an optical system. The half-wave-plate system rotates the incident polarization state such that the reflected wave is polarized exactly in the orthogonal direction. The extent of this orthogonal polarization shift is measured in terms of the PCR:

$$
\eta_{\mathrm{PCR}}=\frac{\left|R_{y x}\right|^{2}}{\left|R_{x x}\right|^{2}+\left|R_{y x}\right|^{2}} .
$$

Therefore, a unit PCR means full rotation of the incident $x$-polarized vectors into the $y$-polarized fields. Similarly, the quarter-wave operation transforms a linearly polarized incident wave into a pure circularly polarized reflected wave. The efficiency of the circular-polarization conversion can be calculated by taking a ratio between the right- $\left(\left|R_{+x}\right|\right)$ and left- $\left(\left|R_{-x}\right|\right)$ handed circular-polarization components. We define the polarization extinction ratio
(PER) as follows:

$$
\eta_{\mathrm{PER}}=20 \log _{10}\left(\frac{\left|R_{+x}\right|}{\left|R_{-x}\right|}\right) .
$$

These two wave-plate behaviors are pointed out in a qualitative manner in the previous section. Here, we numerically deduce the PCR and PER for the proposed metascreen from the surface plots of Fig. 4 so that the type of the associated dichroism can be identified. The extracted polarization parameters are then plotted in Figs. 5(a) and 5(b) for graphene's chemical bias of $600 \mathrm{meV}$. By looking at the PCR and PER plots together, the points of extreme dichroism can be identified. These points are highlighted by the Roman numerals and the corresponding polarization states are plotted in Fig. 5(c). For example, consider the half-power points on the PCR curves, where the magnitudes of the two reflected components are equal. With no phase information associated with PCR, the polarization state cannot be identified. However, since Eq. (9) takes the phase difference into account, the strong $40-\mathrm{dB}$ peaks 

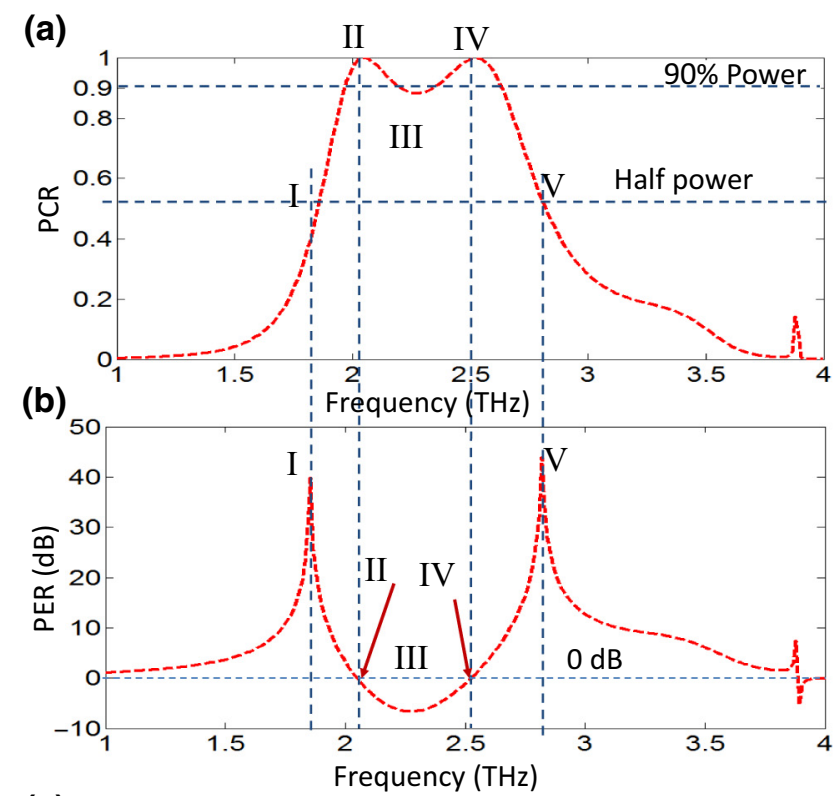

(c)

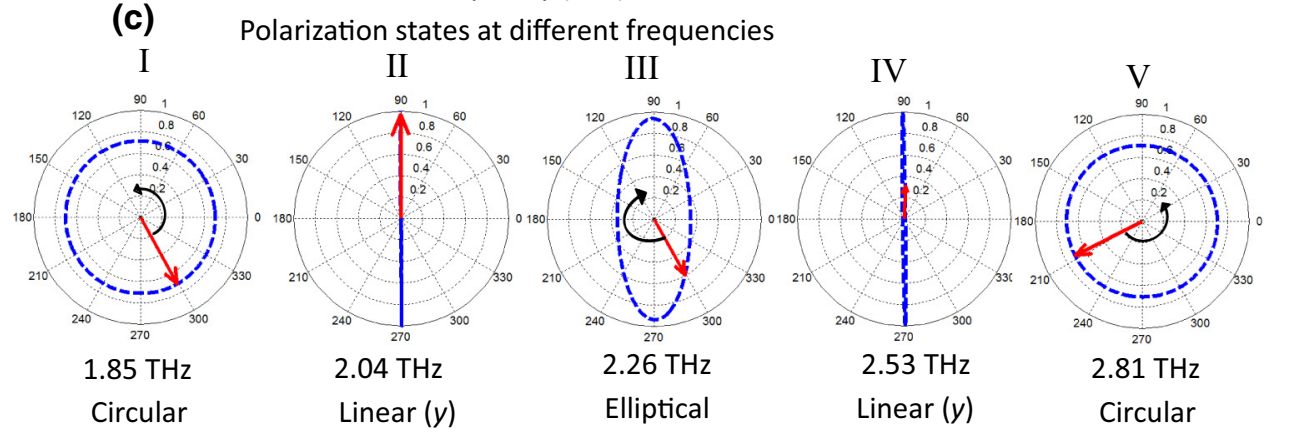

FIG. 5. (a) The PCR for a graphene metascreen. (b) The PER at various frequencies for a fixed chemical potential at 600 $\mathrm{meV}$. (c) The polarization states, showing the tip and direction of rotation of the electric field vector at the frequencies identified in (a) and (b).

in the PER plot point to near-ideal linear-to-circular conversion (quarter-wave-plate behavior). The quarter-waveplate behavior is further established while considering the corresponding polarization-state diagrams (I and V), which show a pure counterclockwise circularly polarized electric field vector.

Let us look further at the spectral points II and IV where the PCR becomes unity, which correspond to strict linear polarized fields as depicted in Fig. 5(c). Here, we appreciate the correspondence between the strictly linearly polarized wave and its PER by considering the linear polarization as a superposition of two equal circularly polarized waves with opposite senses of rotation $\left(\left|R_{+x}\right|\right.$ and $\left.\left|R_{-x}\right|\right)$. Then, it follows from Eq. (9) that these two points attain a 0 -dB PER [Fig. 5(a)]. The PCR remains close to 0.9 in the spectral region between points II and IV, showing that there is a slight contribution of the $x$-component of the field. A small negative value of $-6 \mathrm{~dB}$ PER in this region suggests an elliptically polarized wave, which is indeed confirmed by observing polarization-state diagram III at $2.26 \mathrm{THz}$.

It is worth mentioning that other contemporary metasurfaces also demonstrate similar polarizationconversion capabilities in the electromagnetic spectrum [8-11]. The real strength of the proposed graphene merascreen is its property of reconfigurability, which allows the optical spectrum to be electro-optically tuned. We demonstrate this property by varying the chemical potential of the graphene monolayer from 300 to $800 \mathrm{meV}$, while keeping the frequency fixed at $2.25 \mathrm{THz}$. As depicted in the PER plot of Fig. 6, the polarization state changes from circular for a $360-\mathrm{meV}$ bias to linear at about 416 $\mathrm{meV}$ and then finally to elliptical at $540 \mathrm{meV}$. The reconfigurable graphene chiral monolayer can be potentially exploited in several optical devices, such as optical displays, polarizers, radiating elements, and communication devices.

\section{PARAMETRIC ANALYSIS}

\section{A. Effect of dielectric substrate}

Since the SPP resonances exist in transverse-to-surfacewave propagation, the chiral response of the metascreen is sensitive to resonant electromagnetic interference effects originating from multiple interfaces, i.e., air to graphene surface and substrate to ground surface [30]. Therefore, a parametric study of the variation of the substrate thickness (d) is vital in the chiral metasurface design. Figures 7(a) and 7(b) show the effect of this parameter on the reflection characteristics when $d$ is varied from 100 to $200 \mu \mathrm{m}$. 


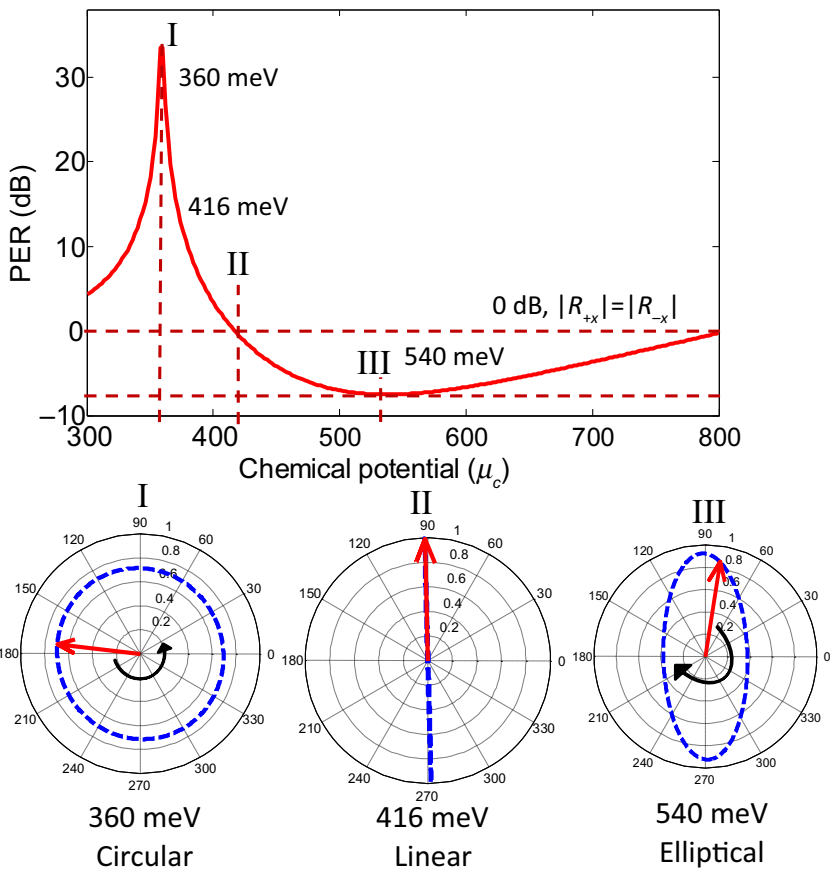

FIG. 6. A demonstration of the tunability. Variation of the polarization states as the chemical potential of the chiral graphene metasurface is changed from 300 to $800 \mathrm{meV}$ at 2.25 THz.

Note the red shift in both the co- $\left(\left|R_{x x}\right|\right)$ and cross- $\left(\left|R_{y x}\right|\right)$ polarization due to the increase in the substrate thickness.

\section{B. Angular stability}

In previous sections, polarization-state control is demonstrated for normal incidence of the light waves. A
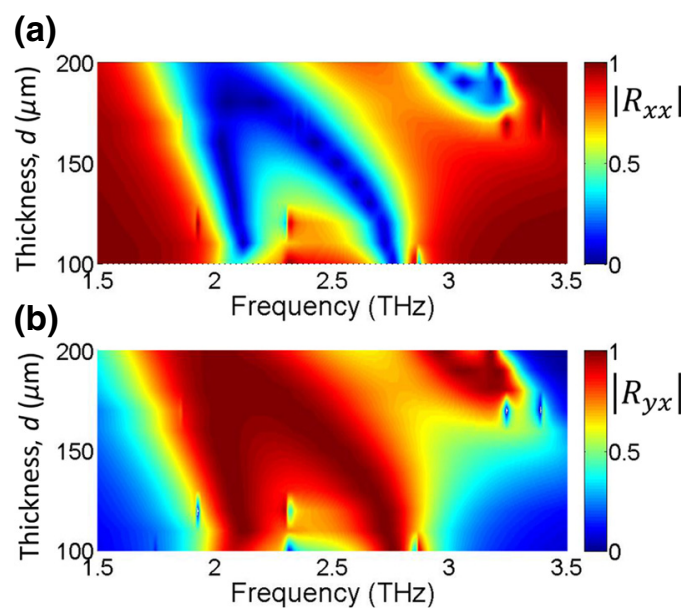

FIG. 7. The response of the graphene metascreen. (a) The co$\left(\left|R_{x x}\right|\right)$ and (b) cross- $\left(\left|R_{y x}\right|\right)$ polarization components due to variation in the substrate height $d$. The color bars are included to represent the scale of the amplitude of the reflection coefficient between 0 to 1 .
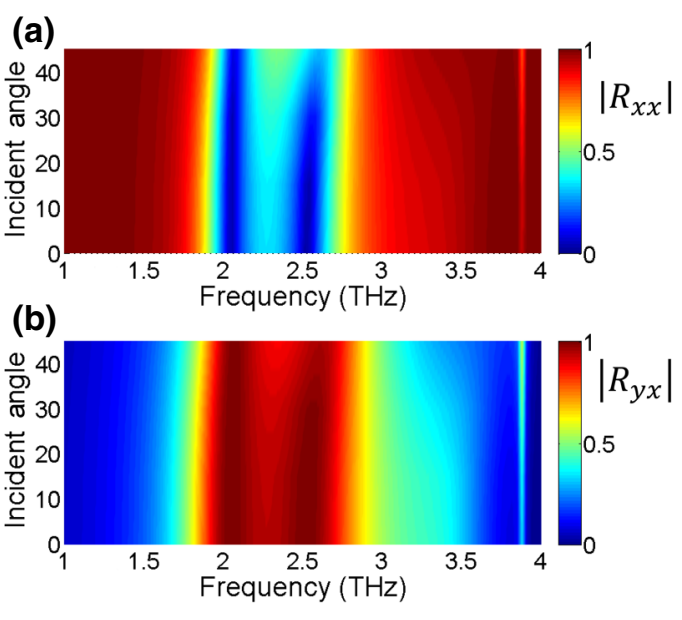

FIG. 8. The angular stability of the graphene metascreen. (a) The co- $\left(\left|R_{x x}\right|\right)$ and (b) cross- $\left(\left|R_{y x}\right|\right)$ polarization components for variation of the incident angle (in degrees) between $0^{\circ}$ and $45^{\circ}$. The color bars are included to represent the scale of the amplitude of the reflection coefficient between 0 and 1 .

reflecting surface is said to have angular stability if the same phenomenon also exists for oblique incidence. Here, we simulate the polarization response of the graphene metascreen when the incident angle is varied between $0^{\circ}$ and $45^{\circ}$. It is emphasized that the incident wave is assumed to be $s$-polarized (having an electric field vector parallel to the metasurface plane). Figure 8 presents the resulting changes in the co- $\left(\left|R_{x x}\right|\right)$ and cross- $\left(\left|R_{y x}\right|\right)$ polarized reflection coefficients. It is clear that the response remains largely stable under oblique incidence, even up to a highly inclined $45^{\circ}$ incidence.

\section{CONCLUSION}

We propose an ultrathin graphene metascreen reflector that exhibits significant optical activity in the terahertz spectrum. The polarization conversion stems from the fact that the helicoidal surface-plasmon current distributions that are formed around the L-shaped unit cells have a chiral symmetry. Instead of depositing L-shaped metal, our design involves etching out L-shaped voids from the metallic layer to form a continuous graphene material that can be conveniently biased by a single electrical circuit. We show a variety of polarization states in the reflection spectrum, ranging from pure linearly dichroic behavior to pure circularly dichroic quarter-wave-plate behavior. For a chemical bias of $600 \mathrm{meV}$, the linear-to-circular polarization conversion is shown at 1.85 and $2.81 \mathrm{THz}$. A strict orthogonal transformation of the linearly polarized incident vector is shown at 2.04- and $2.53-\mathrm{THz}$ bands. In between the two extrema, the graphene metascreen converts the linear states to elliptical polarization states. The most important feature of the chiral metascreen based on graphene is its reconfigurable frequency response, which allows us to tune 
the polarization of the reflected wave to the desired state by varying the Fermi levels. Furthermore, the metascreen reflection response is shown to be angularly stable between $0^{\circ}$ and $45^{\circ}$. The ultracompact unit-cell size, of the order of $\lambda / 15$, makes it suitable for device integration in a myriad of sensing, imaging, and spectroscopy applications at terahertz frequencies.

[1] H. Hecht, Optics (Pearson, London, England, 2017), 5th ed.

[2] Christian Brosseau, Fundamentals of Polarized Light (Wiley, New York, 1998).

[3] A. Lipson, S. Lipson, and H. Lipson, Optical Physics (Cambridge University Press, Cambridge, England, 2010), 4th ed.

[4] Laurence D. Barron, Molecular Light Scattering and Optical Activity (Cambridge University Press, Cambridge, England, 2009).

[5] Indra K. Reddy and Reza Mehvar, Chirality in Drug Design and Development (CRC Press, Florida, US, 2004).

[6] Laurence D. Barron, An Introduction to Chirality at the Nanoscale (Wiley-VCH Verlag GmbH \& Co. KGaA, Weinheim, 2009).

[7] F. Pedrotti, L. Pedrotti, and L. Pedrotti, Introduction to Optics (Pearson, London, England, 2014), 3rd ed.

[8] Muhammad Amin and Adnan D. Khan, Polarization selective electromagnetic-induced transparency in the disordered plasmonic quasicrystal structure, J. Phys. Chem. C 119, 21633 (2015).

[9] Alexander Y. Zhu, Wei Ting Chen, Aun Zaidi, Yao-Wei Huang, Mohammadreza Khorasaninejad, Vyshakh Sanjeev, Cheng-Wei Qiu, and Federico Capasso, Giant intrinsic chiro-optical activity in planar dielectric nanostructures, Light Sci. Appl. 7, 17158 (2018).

[10] Yizhe Zhao, Anyong Qing, Yang Meng, Zelin Song, and Chuan Lin, Dual-band circular polarizer based on simultaneous anisotropy and chirality in planar metamaterial, Sci. Rep. 8, 1729 (2018).

[11] Martin Schäferling, Daniel Dregely, Mario Hentschel, and Harald Giessen, Tailoring Enhanced Optical Chirality: Design Principles for Chiral Plasmonic Nanostructures, Phys. Rev. X 2, 031010 (2012).

[12] Bingnan Wang, Jiangfeng Zhou, Thomas Koschny, Maria Kafesaki, and Costas M. Soukoulis, Chiral metamaterials: Simulations and experiments, J. Opt. A: Pure Appl. Opt. 11, 114003 (2009).

[13] Tuomas Vallius, Konstantins Jefimovs, Jari Turunen, Pasi Vahimaa, and Yuri Svirko, Optical activity in subwavelength-period arrays of chiral metallic particles, Appl. Phys. Lett. 83, 234 (2003).

[14] Adnan Daud Khan and Muhammad Amin, Tunable Salisbury screen absorber using square lattice of plasmonic nanodisk, Plasmonics 12, 257 (2017).

[15] Rashad Ramzan, Muhammad Omar, Omar F. Siddiqui, Muhammad Amin, Nabil Bastaki, and Taoufik Saleh Ksiksi, Electromagnetically induced absorption in the near-field of microwave radiative elements with application to foliage moisture sensing, IEEE Access 6, 77859 (2018).
[16] Meraj-E. Mustafa, Farooq A. Tahir, M. Amin, and O. Siddiqui, Comment on a novel ultrathin and broadband microwave metamaterial absorber [J. Appl. Phys. 116, 094504 (2014)], J. Appl. Phys. 124, 146101 (2018).

[17] Na Liu, Martin Mesch, Thomas Weiss, Mario Hentschel, and Harald Giessen, Infrared perfect absorber and its application as plasmonic sensor, Nano Lett. 10, 2342 (2010).

[18] Muhammad Amin, Mohamed Farhat, and Hakan Bağc1, A nonlinear plasmonic resonator for three-state all-optical switching, Opt. Express 22, 6966 (2014).

[19] Marcus Diem, Thomas Koschny, and Costas M. Soukoulis, Wide-angle perfect absorber/thermal emitter in the terahertz regime, Phys. Rev. B 79, 033101 (2009).

[20] Andreas Tittl, Ann-Katrin U. Michel, Martin Schäferling, Xinghui Yin, Behrad Gholipour, Long Cui, Matthias Wuttig, Thomas Taubner, Frank Neubrech, and Harald Giessen, A switchable mid-infrared plasmonic perfect absorber with multispectral thermal imaging capability, Adv. Mater. 27, 4597 (2015).

[21] Wei Yao, Linlong Tang, Jun Wang, Chunhui Ji Xingzhan Wei, and Yadong Jiang, Spectrally and spatially tunable terahertz metasurface lens based on graphene surface plasmons, IEEE Photonics J. 10, 1 (2018).

[22] M. Amin, O. Siddiqui, W. Orfali, Mohamed Farhat, and A. Khelif, Resonant Beam Steering and Carpet Cloaking Using an Acoustic Transformational Metascreen, Phys. Rev. Appl. 10, 064030 (2018).

[23] M. Amin, O. Siddiqui, Mohamed Farhat, and A. Khelif, A perfect Fresnel acoustic reflector implemented by a Fano-resonant metascreen, J. Appl. Phys. 123, 144502 (2018).

[24] A. V. Rogacheva, V. A. Fedotov, A. S. Schwanecke, and N. I. Zheludev, Giant Gyrotropy Due to Electromagnetic-Field Coupling in a Bilayered Chiral Structure, Phys. Rev. Lett. 97, 177401 (2006).

[25] Justyna K. Gansel, Michael Thiel, Michael S. Rill, Manuel Decker, Klaus Bade, Volker Saile, Georg von Freymann, Stefan Linden, and Martin Wegener, Gold helix photonic metamaterial as broadband circular polarizer, Science $\mathbf{3 2 5}$, 1513 (2009).

[26] Sergei Tretyakov, Igor Nefedov, Ari Sihvola, Stanislav Maslovski, and Constantin Simovski, Waves and energy in chiral nihility, J. Electromagnet. Waves Appl. 17, 695 (2003).

[27] Thierry Verbiest, Guy Koeckelberghs, and Benoît Champagne, Feature issue introduction: Chirality in optics, Opt. Mater. Express 4, 2663 (2014).

[28] Zhijie Ma Yi Li, Yang Li Yandong Gong, Stefan A. Maier, and Minghui Hong, All-dielectric planar chiral metasurface with gradient geometric phase, Opt. Express 26, 6067 (2018).

[29] Zuojia Wang, Feng Cheng, Thomas Winsor, and Yongmin Liu, Optical chiral metamaterials: A review of the fundamentals, fabrication methods and applications, Nanotechnology 27, 412001 (2016).

[30] Muhammad Amin, Mohamed Farhat, and Hakan Bağc1, An ultra-broadband multilayered graphene absorber, Opt. Express 21, 29938 (2013).

[31] M. Amin, Mohamed Farhat, and H. Bagc1, A dynamically reconfigurable Fano metamaterial through graphene tuning 
for switching and sensing applications, Sci. Rep. 3, 2105 (2013).

[32] Zhancheng Li Wenwei Liu, Hua Cheng, Shuqi Chen, and Jianguo Tian, Tunable dual-band asymmetric transmission for circularly polarized waves with graphene planar chiral metasurfaces, Opt. Lett. 41, 3142 (2016).

[33] Junyang Zhao, Jianfa Zhang, Zhihong Zhu, Xiaodong Yuan, and Shiqiao Qin, Tunable asymmetric transmission of $\mathrm{THz}$ wave through a graphene chiral metasurface, J. Opt. 18, 095001 (2016).

[34] Huan Jiang, Wenyu Zhao, and Yongyuan Jiang, Highefficiency tunable circular asymmetric transmission using dielectric metasurface integrated with graphene sheet, Opt. Express 25, 19732 (2017).

[35] Frank H. L. Koppens, Darrick E. Chang, and F. Javier Garcia de Abajo, Graphene plasmonics: A platform for strong light-matter interactions, Nano Lett. 11, 3370 (2011).

[36] Kostya S. Novoselov, Andre K. Geim, Sergei V. Morozov, D. Jiang, Y. Zhang, Sergey V. Dubonos, Irina V. Grigorieva, and Alexandr A. Firsov, Electric field effect in atomically thin carbon films, Science 306, 666 (2004).

[37] Andre K. Geim and Konstantin S. Novoselov, in Nanoscience and Technology: A Collection of Reviews from Nature Journals (World Scientific, 2010) pp. 11-19.

[38] A. H. Castro Neto, Francisco Guinea, Nuno M. R. Peres, Kostya S. Novoselov, and Andre K. Geim, The electronic properties of graphene, Rev. Mod. Phys. 81, 109 (2009).

[39] Long Ju, Baisong Geng, Jason Horng, Caglar Girit, Michael Martin, Zhao Hao, Hans A. Bechtel, Xiaogan Liang, Alex Zettl, and Y. Ron Shen, et al., Graphene plasmonics for tunable terahertz metamaterials, Nat. Nanotechnol. 6, 630 (2011).

[40] A. N. Grigorenko, Marco Polini, and K. S. Novoselov, Graphene plasmonics, Nat. Photonics 6, 749 (2012).

[41] Vinit Singh Yadav, Sambit Kumar Ghosh, Somak Bhattacharyya, and Santanu Das, Graphene-based metasurface for a tunable broadband terahertz cross-polarization converter over a wide angle of incidence, Appl. Opt. 57, 8720 (2018).

[42] Tianjing Guo and Christos Argyropoulos, Broadband polarizers based on graphene metasurfaces, Opt. Lett. 41, 5592 (2016).

[43] Stefan Alexander Maier, Plasmonics: Fundamentals and Applications (Springer Science \& Business Media, Berlin, Germany, 2007).

[44] Gururaj V. Naik, Vladimir M. Shalaev, and Alexandra Boltasseva, Alternative plasmonic materials: Beyond gold and silver, Adv. Mater. 25, 3264 (2013).

[45] George W. Hanson, Quasi-transverse electromagnetic modes supported by a graphene parallel-plate waveguide, J. Appl. Phys. 104, 084314 (2008).
[46] Jianing Chen, Michela Badioli, Pablo Alonso-González, Sukosin Thongrattanasiri, Florian Huth, Johann Osmond, Marko Spasenović, Alba Centeno, Amaia Pesquera, and Philippe Godignon, et al., Optical nano-imaging of gatetunable graphene plasmons, Nature 487, 77 (2012).

[47] Ruitao Lv, Qing Li, Andrés R. Botello-Méndez, Takuya Hayashi, Bei Wang, Ayse Berkdemir, Qingzhen Hao, Ana Laura Elías, Rodolfo Cruz-Silva, and Humberto R. Gutiérrez, et al., Nitrogen-doped graphene: Beyond single substitution and enhanced molecular sensing, Sci. Rep. 2, 586 (2012).

[48] Rasoul Alaee, Mohamed Farhat, Carsten Rockstuhl, and Falk Lederer, A perfect absorber made of a graphene micro-ribbon metamaterial, Opt. Express 20, 28017 (2012).

[49] Pai-Yen Chen, Mohamed Farhat, and Hakan Bağcı, Graphene metascreen for designing compact infrared absorbers with enhanced bandwidth, Nanotechnology 26, 164002 (2015).

[50] Pai-Yen Chen, Christos Argyropoulos, Mohamed Farhat, and J. Sebastian Gomez-Diaz, Flatland plasmonics and nanophotonics based on graphene and beyond, Nanophotonics 6, 1239 (2017).

[51] Mohamed Farhat, Sébastien Guenneau, and Hakan Bağc1, Exciting Graphene Surface Plasmon Polaritons through Light and Sound Interplay, Phys. Rev. Lett. 111, 237404 (2013).

[52] Pai-Yen Chen and Andrea Alu, A terahertz photomixer based on plasmonic nanoantennas coupled to a graphene emitter, Nanotechnology 24, 455202 (2013).

[53] Farzad Zangeneh-Nejad and Reza Safian, A graphenebased THz ring resonator for label-free sensing, IEEE Sens. J. 16, 4338 (2016).

[54] Triranjita Srivastava, Amrita Purkayastha, and Rajan Jha, Graphene based surface plasmon resonance gas sensor for terahertz, Opt. Quant. Electron. 48, 334 (2016).

[55] Farzad Zangeneh-Nejad and Reza Safian, Hybrid graphenemolybdenum disulphide based ring resonator for label-free sensing, Opt. Commun. 371, 9 (2016).

[56] Andrea C. Ferrari, J. C. Meyer, Vittorio Scardaci, Cinzia Casiraghi, Michele Lazzeri, Francesco Mauri, Stefano Piscanec, Da Jiang, K. S. Novoselov, and S. Roth, et al., Raman Spectrum of Graphene and Graphene Layers, Phys. Rev. Lett. 97, 187401 (2006).

[57] Kaveh Khaliji, Sudipta Romen Biswas, Hai Hu Xiaoxia Yang, Qing Dai, Sang-Hyun Oh Phaedon Avouris, and Tony Low, Plasmonic Gas Sensing with Graphene Nanoribbons, Phys. Rev. Appl. 13, 011002 (2020).

[58] F. Falcone, T. Lopetegi, M. A. G. Laso, J. D. Baena, J. Bonache, M. Beruete, R. Marqués, Ferran Martín, and M. Sorolla, Babinet Principle Applied to the Design of Metasurfaces and Metamaterials, Phys. Rev. Lett. 93, 197401 (2004). 